\title{
Constraining a possible time-variation of the gravitational constant through "gravitochemical heating" of neutron stars
}

\author{
Andreas Reisenegger ${ }^{1}$, Paula Jofré ${ }^{2}$ and Rodrigo Fernández ${ }^{3}$ \\ ${ }^{1}$ Depto. de Astronomía y Astrofísica, Pontificia Universidad Católica de Chile, \\ Santiago, Chile \\ email: areisene@astro.puc.cl \\ ${ }^{2}$ Max-Planck-Institut für Astrophysik, Garching, Germany \\ ${ }^{3}$ Department of Astronomy and Astrophysics, University of Toronto, Toronto, Canada \\ Present address: Institute for Advanced Study, Princeton, USA
}

\begin{abstract}
A hypothetical time-variation of the gravitational constant $G$ would produce gravitochemical heating of old ( $\gtrsim 10^{7-8} \mathrm{yr}$ ) neutron stars. It could produce detectable thermal ultraviolet emission from such stars for changes as small as $|\dot{G} / G| \sim 10^{-12} \mathrm{yr}^{-1}$, comparable to the best existing upper limits from other methods.
\end{abstract}

Keywords. Stars: Neutron, Dense matter, Gravitation, Relativity, Pulsars: general, Pulsars: individual (PSR J0437-4715), Ultraviolet: stars

If the gravitational constant $G$ is not strictly constant, as hypothesized by many authors since Dirac (1937), neutron stars would expand or contract, so the mix of degenerate fermions in their interiors would slightly depart from beta equilibrium. This induces non-equilibrium beta processes (direct or modified Urca reactions), such as the neutron beta decay $(n \rightarrow p+e+\bar{\nu})$ and its inverse $(p+e \rightarrow n+\nu)$, which release energy that is invested partly in neutrino emission and partly in heating the star. After $\sim 10^{7-8} \mathrm{yr}$, the star arrives at a stationary state in which the temperature remains nearly constant, as the forcing through the change of $G$ is balanced by the ongoing reactions. Using the surface temperature $\left(\sim 10^{5} \mathrm{~K}\right)$ of the nearest millisecond pulsar, PSR J0437-4715, inferred from ultraviolet observations with the Hubble Space Telescope (Kargaltsev et al. 2004), we have estimated two upper limits for this variation (Jofré et al. 2006): (1) $|\dot{G} / G|<2 \times 10^{-10} \mathrm{yr}^{-1}$, if direct Urca reactions are allowed, and (2) $|\dot{G} / G|<4 \times 10^{-12} \mathrm{yr}^{-1}$, considering only modified Urca reactions. The latter is among the most restrictive obtained by other methods (e. g., Reisenegger 2007). Further study of neutron star physics and additional observations of old neutron stars should be able to refine these constraints.

\section{Acknowledgements}

This work was supported by FONDECYT Regular Grant 1060644, the FONDAP Center for Astrophysics, and the Basal Funding Project PFB-06/2007.

\section{References}

Dirac, P. 1937, Nature, 139, 323

Jofré, P., Reisenegger, A., \& Fernández, R. 2006, Phys. Rev. Lett., 97, 131102

Kargaltsev, O., Pavlov, G. G., \& Romani, R. 2004, ApJ, 602, 327

Reisenegger, A., Fernández, R., \& Jofré, P., 2006, Ap. Sp. Sci., 308, 413 